\title{
A Case of Prostate Carcinoma Discovered With Pulmonary Lymphangitis Carcinomatosa
}

\author{
Hiroshi Ono ${ }^{\mathrm{a}, \mathrm{e}}$, Yasuyuki Taniguchi ${ }^{\mathrm{a}}$, Yoshinobu Komai ${ }^{\mathrm{b}}$, Masashi Kawamoto ${ }^{\mathrm{c}}$, \\ Hiroki Hayashi $^{\mathrm{d}}$, Akihiko Gemma ${ }^{\mathrm{d}}$
}

\begin{abstract}
A 68-year-old man suffering from dyspnea since mid December 2009 presented at our hospital in the beginning of January 2010 because of gradual worsening of the problem. In chest X-ray and computed tomography (CT), the density existed in peribronchovascular and peripheral interstitium. This was followed by a transbronchial lung biopsy (TBLB), which led to the suspicion of a lymphagitis carcinomatosa. In the systemic CT, the prostate was found enlarged. Following a prostate biopsy, the final diagnosis was prostate cancer. This case is reported because of the rarity for a prostate carcinoma to be discovered with pulmonary lymphangitis carcinomatosa.
\end{abstract}

Keywords: Lympohangitis carcinomatosa; Prostate cancer; Transbronchial lung biopsy (TBLB)

\section{Introduction}

It is rare for prostate cancer (PC) to be discovered with an abnormality of chest X-ray as the initial finding. According to Sugama and coworkers, only 37 such cases were reported by 2003 in Japan [1]. Of course, those cases discovered with pulmonary lymphangitis carcinomatosa (LC) are much rarer. We now report a case of prostate cancer which was noted first as an abnormality in chest X-ray and was diagnosed

Manuscript accepted for publication February 4, 2011

${ }^{a}$ Department of Internal Medicine, Jiseikai-Tojun Hospital, Japan

${ }^{\mathrm{b}}$ Department of Urology, Jiseikai-Tojun Hospital, Japan

${ }^{\mathrm{c} D}$ Department of Analytic Human Pathology, Nippon Medical School,

Japan

${ }^{\mathrm{d}}$ Division of Pulmonary Medicine, Infectious Disease, and Oncology,

Department of Internal Medicine, Nippon Medical School, Japan

${ }^{\mathrm{e}}$ Corresponding author: Hiroshi Ono, email: h-ono@nms.ac.jp

doi:10.4021/jmc148w as pulmonary lymphoangitis carcinomatosa with a transbronchial lung biopsy (TBLB). Finally, prostate cancer was discovered with a computed tomography (CT) scan and a prostate biopsy.

\section{Case Report}

The case was a 68-year-old man whose occupation was an independent businessman who engaged mainly in desk work. His chief complaints were abnormality in chest X-ray, dyspnea, general fatigue and pyrexia. He was noted for hypertension and diabetes mellitus in a prior screening survey, but did not have a checkup at a medical institution because of his reluctance to see a physician. Dyspnea developed since the middle of December 2009, which was ignored. However, due to gradual worsening, he was examined at a nearby clinic in the beginning of January 2010. In the clinic, abnormalities were seen in chest X-rays and he was recommended to have further studies at our hospital. He had no special family history, no inhalation history and no allergy. As for luxury, he was a current smoker who had been smoking 1.5 packs of cigarettes per day for 48 years, and drank a can $(350 \mathrm{ml})$ of beer per day.

In initial diagnosis, His consciousness was alert. Body temperature was 37.9 degrees centigrade. Pulse was 133 per minute regular. Blood pressure was 173/95. Oxygen saturation was $95 \%$. There was no adventitious sound in auscultation and no palpable superficial lymph nodes in palpating. In the blood examination, there was mild leukocytosis and mild liver dysfunction, and levels of lactate dehydrogenase (LDH), C reactive protein (CRP), blood glucose and hemoglobin $\mathrm{A}_{1 \mathrm{c}}\left(\mathrm{HbA}_{1 \mathrm{c}}\right)$ were remarkably high. And in blood gas analysis, there was a mild hypocapnemia (Table 1). On the chest X-ray, there was diffuse, bilateral ground glass opacity and lung outline was thickened irregularly (Fig. 1), and on the high resolution computed tomography (HRCT), ground glass and granular opacities were present diffusely around bronchovascular bundles, and peribronchovascular interstitial thickenings and interlobular septa thickenings which form polygons were present in spots. In mediastinal spaces, some mediastinal lymph nodes were slightly enlarged (Fig. 
Table 1. Blood Examination and Blood Gas Analysis

\begin{tabular}{|c|c|c|c|c|c|}
\hline CBC & & Normal range & & & Normal Range \\
\hline WBC & $12600 / \mu \mathrm{l}$ & $(3900-9300)$ & Cre & $0.92 \mathrm{mg} / \mathrm{dl}$ & \\
\hline $\mathrm{RBC}$ & $511 \times 10^{4} / \mu 1$ & & $\mathrm{Na}$ & $141 \mathrm{mEq} / \mathrm{L}$ & \\
\hline hemoglobin & $14.5 \mathrm{~g} / \mathrm{dl}$ & & $\mathrm{K}$ & $4.0 \mathrm{mEq} / \mathrm{L}$ & \\
\hline hematocrit & $43.6 \%$ & & $\mathrm{Cl}$ & $99 \mathrm{mEq} / \mathrm{L}$ & $(70-109)$ \\
\hline platelet & $19 \times 10^{4} / \mu \mathrm{l}$ & & Glu & $221 \mathrm{mg} / \mathrm{dl}$ & $(4.3-5.8)$ \\
\hline Hemogram & & & $\mathrm{HbA}_{1 \mathrm{c}}$ & $8.5 \%$ & $(<0.3)$ \\
\hline neutrophil & $76 \%$ & & CRP & $28.4 \mathrm{mg} / \mathrm{dl}$ & \\
\hline lymphocyte & $16.8 \%$ & & CEA & $0.5 \mathrm{ng} / \mathrm{ml}$ & \\
\hline monocyte & $6.3 \%$ & & Ca 19-9 & $11.1 \mathrm{U} / \mathrm{ml}$ & $(124-466)$ \\
\hline eosinophil & $0.3 \%$ & & SIL2R & $564 \mathrm{U} / \mathrm{ml}$ & \\
\hline basophil & $0.6 \%$ & & KL-6 & $257 \mathrm{U} / \mathrm{ml}$ & \\
\hline BBC & & & SP-D & $17.2 \mathrm{ng} / \mathrm{ml}$ & \\
\hline T-Bil & $1.9 \mathrm{mg} / \mathrm{dl}$ & $(0.2-1.0)$ & PR3-ANCA & $<10 \mathrm{EU}$ & \\
\hline AST & $95 \mathrm{U} / \mathrm{L}$ & $(5-40)$ & MPO-ANCA & $<10 \mathrm{EU}$ & \\
\hline ALT & $48 \mathrm{U} / \mathrm{L}$ & $(5-45)$ & ABG & & \\
\hline LDH & $1293 \mathrm{U} / \mathrm{L}$ & $(120-240)$ & $\mathrm{PH}$ & 7.464 & \\
\hline$\gamma$-GTP & $187 \mathrm{U} / \mathrm{L}$ & $(\leq 85)$ & $\mathrm{PCO}_{2}$ & $31 \mathrm{~mm} \mathrm{Hg}$ & $(32-46)$ \\
\hline CPK & $133 \mathrm{U} / \mathrm{L}$ & & $\mathrm{PO}_{2}$ & $79 \mathrm{~mm} \mathrm{Hg}$ & \\
\hline ТP & $6.8 \mathrm{~g} / \mathrm{dl}$ & & $\mathrm{HCO}_{3}^{-}$ & $22.0 \mathrm{mmol} / \mathrm{L}$ & \\
\hline UA & $4.5 \mathrm{mg} / \mathrm{dl}$ & & $\mathrm{BE}$ & $-0.6 \mathrm{mmol} / \mathrm{L}$ & \\
\hline BUN & $25.9 \mathrm{mg} / \mathrm{dl}$ & $(8.0-20.0)$ & $\mathrm{SaO}_{2}$ & $96.9 \%$ & \\
\hline
\end{tabular}

CBC, complete blood count; WBC, white blood cell; RBC, red blood cell; BBC, blood biochemistry; AST, aspartate aminotransferase; ALT, alanine aminotransferase; LDH, lactate dehydrogenase; $\gamma$-GTP, $y$-glutamyltranspeptidase; CPK, creatine phosphate kinase; TP, total protein; UA, uric acid; BUN, blood urea nitrogen; Cre, creatinine; Glu, glucose; $\mathrm{HbA}_{10}$, hemoglobin $\mathrm{A}_{1 \mathrm{c}}$; $\mathrm{CRP}, \mathrm{C}$ reactive protein; CEA, carcinoembryonic antigen; sLI-2R, serum interleukin II receptor; PR3, proteinase-3; ANCA, antineutrophil cytoplasmic antibody; MPO, myeloperoxidase; $\mathrm{ABG}$, arterial blood gas analysis; $\mathrm{BE}$, base excess; $\mathrm{SaO}_{2}$, Oxygen saturation.

2). In the sputum culture, only indigenous bacteria were detected. Both acid-fast bacillus smears and the cultures were negative. In the gastric juice analysis, both acid-fast bacillus smears and cultures were negative.

From pyrexia, leucocytosis, and high level of CRP, some types of infectious pulmonary disease were suspected initially, and azithromycin and levofloxacin were administered. Although the efficacy of these drugs was uncertain, his body temperature and symptoms improved, and the number of WBC's and CRP level improved to 9900 and 9.46 respectively by 6 days after the initial diagnosis. However, the findings of chest X-ray and CT remained the same. Therefore, a fiberoptic bronchoscopy (FB) was performed in the middle of January 2010. The observation of the bronchial lumen indicated no bronchial morphological abnormality and as for bronchoalveolar lavage (BAL) from left B5b, it was judged 


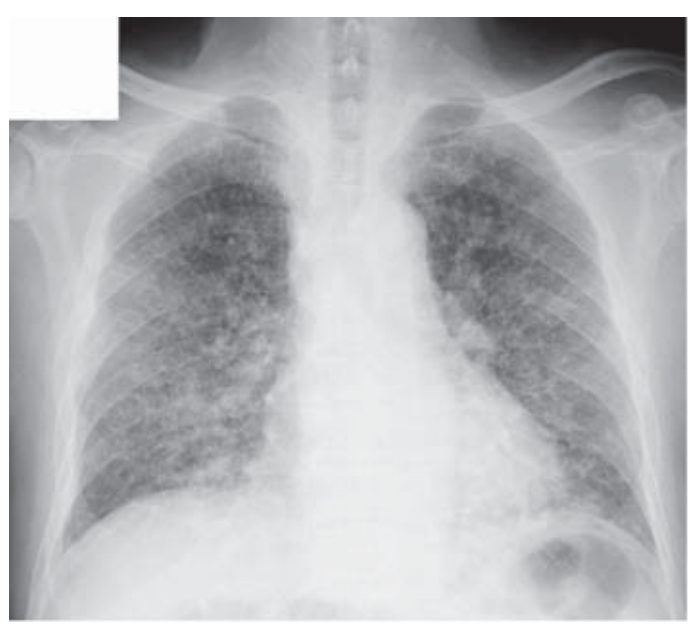

Figure 1. The chest X-ray in the initial diagnosis.

as Class I in the cytology and no bacteria, including acid-fast bacilli, were detected. In the third lavage of BAL, quantity of collection was $31 \mathrm{ml} / 50 \mathrm{ml}$, cell count was $6.3 \times 10^{5} / \mathrm{ml}$ and among cells, the ratio of macrophage was $82 \%$, lymphocyte was $14 \%$, neutrophil was $4 \%$ and eosinophil was $0 \%$. Furthermore, we performed trans-bronchial lung biopsy (TBLB) which was obtained from B8a, B4a, and B3a in the left lung. In Hematoxylin and Eosin staining of the specimens of TBLB, cell colonies were observed whose origin was unknown, and where nuclei were slightly enlarged, with prominent nucleoli (Fig. 3). Because an infiltrate to lymph ducts was present when we performed anti-lymph duct endodermis antibody (D2-40) staining for these, lymphangitis carcinomatosa (LC) was suspected. Since the specimen was not stained positive with thyroid transcription factor 1(TTF1), it was clear that the origin would not be lung (Fig. 4). However, the origin of the primary lesion was not estab-

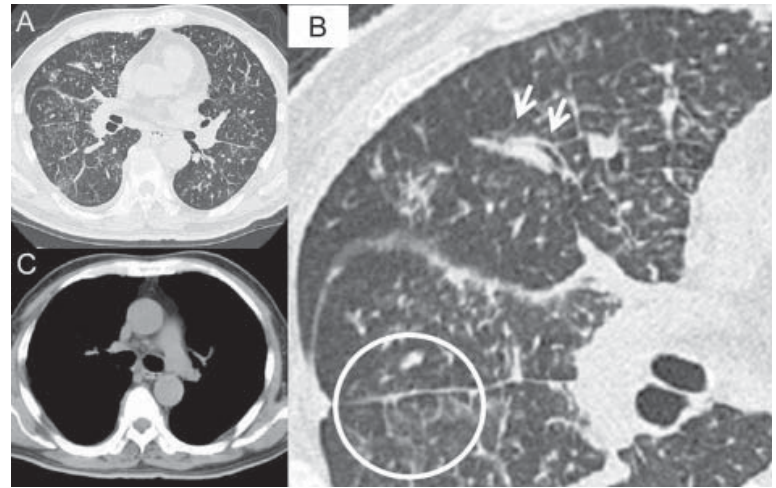

Figure 2. High resolution computed tomography (HRCT) of the chest in the initial diagnosis. In the lung fields $(A)$, ground glass and granular opacities are present diffusely around bronchovascular bundle. In the macrophotograph of the right middle lobe and part of lower lobe (B), peribronchovascular interstitial thickening (arrows) and interlobular septa thickening which form a polygon (in the circle) are present. In the mediastinal space condition (C), some mediastinal lymph nodes are slightly enlarged.

lished.

FDG-PET could not be performed because the patient had diabetes mellitus. Thus, endoscopy of the intestinal tract (stomach, colon) was performed as well as enhanced systemic CT in searching for the original lesion. In gastric tract fiberscopy, a duodenal polyp was observed which was benign in biopsy, and colonoscopy showed only a sigmoid diverticulum. However, systemic enhanced CT showed a remarkably enlarged prostate where the interior density was heterogeneous (Fig. 5). The level of serum prostate-specific antigen (PSA) measured at the same time was remarkably high $(11,149 \mathrm{ng} / \mathrm{ml})$. Later, when PSA staining was performed by the pathology unit, staining was positive in the area of colonization of atypical cells (Fig. 6). In addition,
$(10 X)$

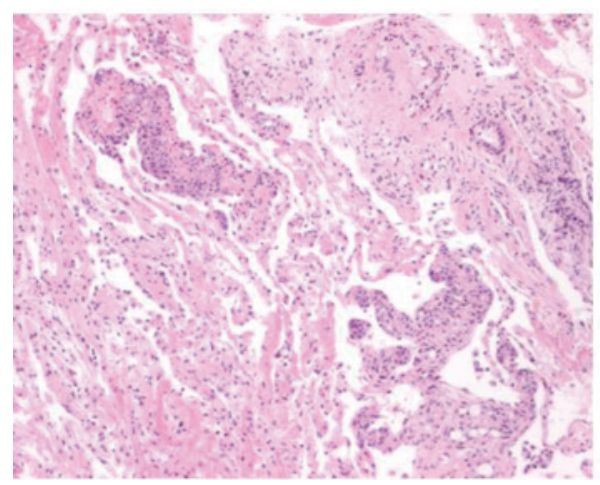

$(40 X)$

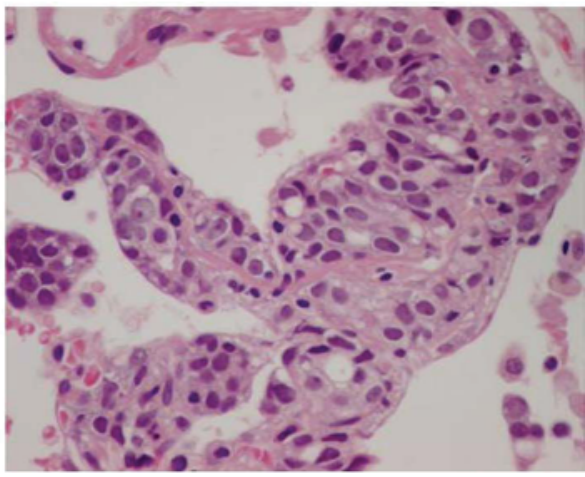

Figure 3. The specimen of trans-bronchial lung biopsy (TBLB) with Hematoxylin and Eosin staining. 
TTF-1 staining $(20 \mathrm{X})$

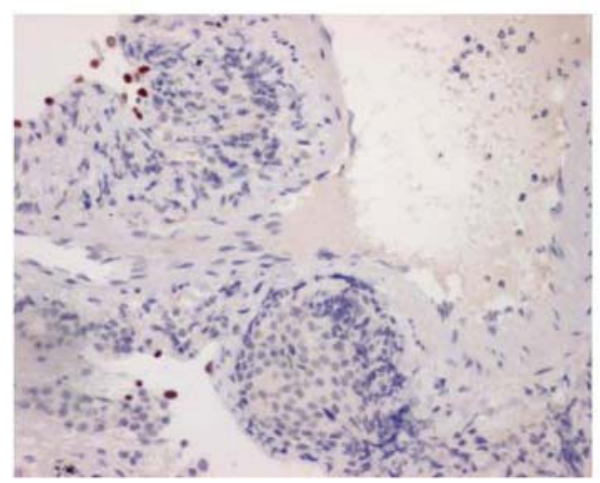

D2-40 staining $(20 \mathrm{X})$

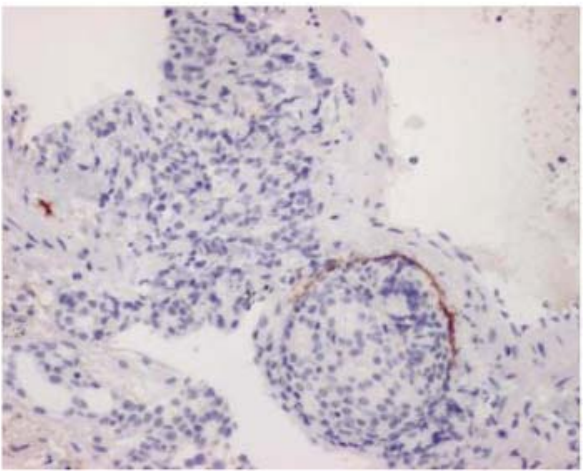

Figure 4. The specimens of TBLB with TTF-1 staining (left) and D2-40 staining (right).

a biopsy revealed a poorly differentiated adenocarcinoma with Gleason's score of eight (Fig. 7). Therefore, the chest radiological abnormality was diagnosed definitively as LC of prostatic origin. For treatment, an oral androgenic agent (bicalutamide $80 \mathrm{mg}$ per day) was administered in the outpatient department of urology in our hospital since the beginning of March. Eleven days later, the PSA value increased to $15,432 \mathrm{ng} / \mathrm{ml}$, but for two weeks after the initiation, the appetite of the patient gradually improved and general fatigue was abated. This enabled him to go out, and the treatment was continued. A combination treatment with goserelin (LH-RH analog agent) was planned for four weeks after the initial regimen. However, in the end of March, twenty-one days after the initiation, dyspnea developed suddenly when he drank water at daybreak, and he was urgently admitted to our hospital. In the chest X-ray and CT, permeability of lung fields decreased remarkably and bilateral pleural effusions developed. Although pulse therapy with methylprednisolone

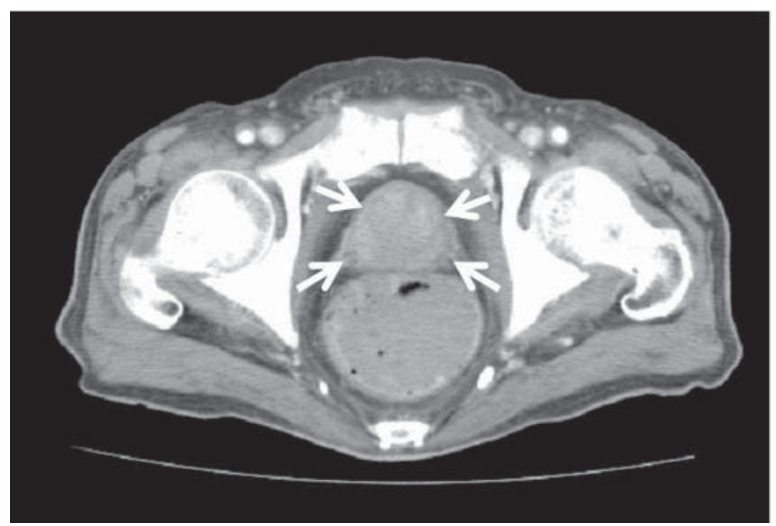

Figure 5. Pelvic CT showing a remarkably enlarged prostate with heterogeneous interior density. and intravenous morphine hydrochloride was given for reduction of dyspnea, his condition did not improve, and he expired three days after the hospitalization.

\section{Discussion}

LC is the state in which cancer cells infiltrate intrapulmonary broad lymph ducts and form emboli. High resolution CT studies of LC have reported the following findings: interlobular septal thickening of a polygon (form of carapace), centrilobular granular lesion, branching opacities and smooth or irregular peribronchovascular interstitial thickening [2]. But it is assumed there are no pulmonary structural distortions [3]. A lymphoproliferative disorder or sarcoidosis is considered for the differential diagnosis. The findings mentioned

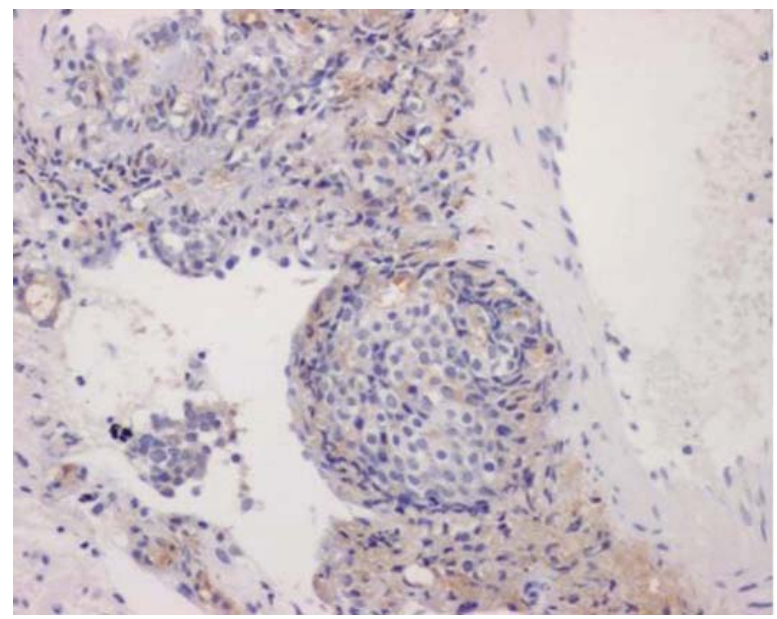

Figure 6. PSA staining was positive in the area of colonization of atypical cells $(10 \mathrm{X})$. 
$(20 \mathrm{X})$

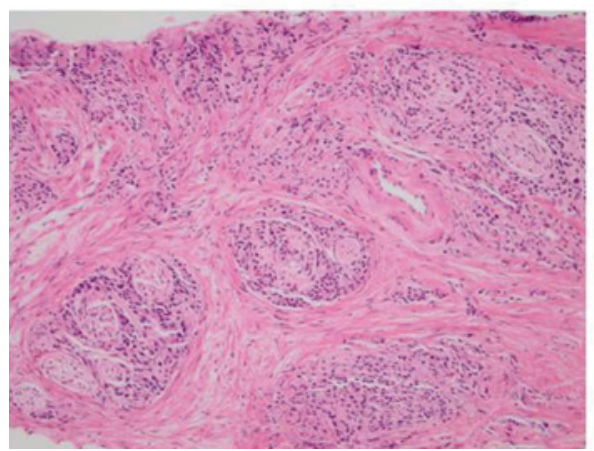

$(40 \mathrm{X})$

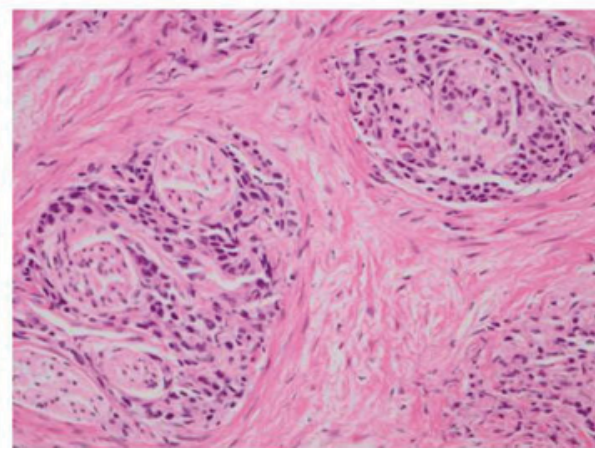

Figure 7. The prostate biopsy showing a poorly differentiated adenocarcinoma and in which Gleason's score was 8.

above are almost consistent with the CT findings of our present case. For a confirmation of diagnosis, it is necessary to perform cytology of sputa, or specimens obtained with BF, or histopathological diagnosis of a lung biopsy. Levy reviewed the diagnosis rate of $\mathrm{LC}$ with $\mathrm{FB}$ and reported that the BAL showed a diagnosis rate of $100 \%$ in comparison with $44 \%$ for TBLB [4]. However, in the present case, the cytology of BAL was negative and LC was diagnosed for the first time with TBLB. Moreover, Asano and coworkers reported that for diagnosis of $\mathrm{LC}$ of a breast cancer origin, although the cytology of BAL was negative LC was similarly diagnosed with TBLB [5]. Therefore, it is recommended that TBLB is performed as well as BAL as much as possible for a diagnosis of LC.

According to the investigation by Yang and his coworkers in 1972, among 275 cases of LC including 62 of their own cases and reports of the past, the most frequent sites of origin were: stomach (122 cases); bronchus (62 cases), breast ( 25 cases), pancreas (14 cases), and uterus (11 cases). These were followed by 10 cases each of prostate and colon [6]. Thus, the prostate accounts for only a small fraction as a primary organ of LC. On the other hand, according to past investigations, pleural effusions had the highest frequency $(22 \%)$ among radiologic findings of pulmonary metastasis from prostate carcinoma, followed by nodular lesions $(8 \%)$, lymphoadenopathy (4.5\%), and reticulo-nodular lesions $(3.5 \%)$ [7]. The portion means that reticular or reticulo-nodular lesion could be interpreted as findings of LC. It could be said that LC in prostate carcinoma is not rare at all because if both findings are combined, it would amount to a total of $20 \%$ of pulmonary abnormalities in prostate carcinoma. However, there were about $4.9 \%-6.7 \%$ of clinical abnormalities in lungs that were found among cases clinically diagnosed as a PC [8-10] although pathological changes in the lungs were found in $12 \%-38 \%$ among autopsied cases of prostate carcinoma according to past reports $[11,12]$. Conceivably, the reason for the difference between both sides is the urologist and the pulmonologist both ignore the association of the prostate and lungs. It is necessary that the potential of the prostate as a primary organ is considered when an image is detected in lungs suspicious of a carcinomatous metastasis. In the male patient, on the other hand, the likelihood of spreading to the lungs is always assumed when prostatic cancer is detected.

In general, multiple spread of carcinoma to the lung including $\mathrm{LC}$ is a form of the terminal phase of carcinoma and leads to a poor prognosis. However, only for prostate carcinoma, some studies have reported remarkably effective use of maximum androgen blockade (MAB) therapy (the combination of anti-androgenic agent and LH-RH analog agent) [13-15]. Therefore in the present case, MAB therapy was appropriate although his condition aggravated rapidly and death ensued. Because an autopsy was not performed in the present case, the exacerbating cause of the CT images of the lungs just before death could not be determined. However, the PSA level was increased eleven days after the initiation of bicalutamide. This could mean that the patient's carcinoma was a poor responder to hormonotherapy and the result would be the exacerbation of LC itself. It should be recognized that although prostate carcinoma may respond to treatment, it may have progressed considerably when multiple lesions were observed in lungs, and that in spite of treatments, a patient might follow a miserable course as described in the present case.

\section{References}

1. Sugama Y, Uji M, Matsushita H. [Two cases of prostate cancer found primarily from lung metastatic lesions]. Nihon Kokyuki Gakkai Zasshi 2003;41(10):733-738.

2. Johkoh T, Ikezoe J, Tomiyama N, Nagareda T, Kohno $\mathrm{N}$, Takeuchi N, Yamagami H, et al. CT findings in lymphangitic carcinomatosis of the lung: correlation with 
histologic findings and pulmonary function tests. AJR Am J Roentgenol 1992;158(6):1217-1222.

3. Paslawski M, Krzyzanowski K, Zlomaniec J. Lymphangitis carcinomatosa in thin section computed tomography. Ann Univ Mariae Curie Sklodowska Med 2004;59(1):1-5.

4. Levy H, Horak DA, Lewis MI. The value of bronchial washings and bronchoalveolar lavage in the diagnosis of lymphangitic carcinomatosis. Chest 1988;94(5):10281030.

5. Asano M, Matsuo K, Ueda Y, Tsushima M, Mizuta M, Fujiwara K, Yonei T, et al. [Pulmonary carcinomatous lymphangiosis from recurrent breast cancer 10 years after resection of the primary tumor]. Nihon Kokyuki Gakkai Zasshi 2009;47(7):602-607.

6. Yang SP, Lin CC. Lymphangitic carcinomatosis of the lungs. The clinical significance of its roentgenologic classification. Chest 1972;62(2):179-187.

7. Wu JW, Chiles C. Lymphangitic carcinomatosis from prostate carcinoma. J Comput Assist Tomogr 1999;23(5):761-763.

8. Petersen RO. Prostate in Petersen RO (ed) Urological
Pathology. Philadelphia, Lippincott, 1992;589-667.

9. Elkin M, Mueller HP. Metastases from cancer of the prostate; autopsy and roentgenological findings. Cancer 1954;7(6):1246-1248.

10. Bolton BH. Pulmonary Metastases from Carcinoma of the Prostate: Incidence and Case Report of a Long Remission. J Urol 1965;94:73-77.

11. Bunpus HC jr. Carcinoma of the prostate; clinical study of 1000 cases. Surg Gynecol Obstet 1926;43:150-155.

12. Mintz ER and Smith GE. Autopsy finding in 100 cases of prostate cancer. N Engl J Med 1934;211:479-487.

13. Fukuda M, Takashima H, Fuse H, Hirano S. [Prostatic cancer with multiple pulmonary metastases treated successfully with hormonal therapy: a case report]. Hinyokika Kiyo 2002;48(8):499-502.

14. Fabozzi SJ, Schellhammer PF, el-Mahdi AM. Pulmonary metastases from prostate cancer. Cancer 1995;75(11):2706-2709.

15. Morin ME, MacNealy GA, Tan A, Li YP, Engel G, Henneberry M. Hormonal manipulation of pulmonary metastases from carcinoma of the prostate. Urol Radiol 1982;4(1):23-27. 